\section{Reparación de válvula mitral y cierre de comunicación interauricular, asistida por robot: experiencia inicial}

\author{
EDGARDO SEPÚLVEDA ${ }^{1}$, ANÍBAL IBÁÑEZ ${ }^{1}$, CRISTIAN BAEZA $^{1}$, \\ MANUEL ESPÍNDOLA ${ }^{1}$, GUSTAVO SEPÚLVEDA ${ }^{1}$, \\ MAURICIO MAUREIRA ${ }^{1}$, JUAN PABLO URIBE ${ }^{1}$, CRISTIAN SALAS ${ }^{1}$
}

\section{Robotic mitral valve repair and closure of atrial septal defect. Report of 13 procedures}

Background: Robot-assisted minimally invasive heart surgery is an effective alternative when compared with classical approaches. It has a low mortality and postoperative complications and its long-term durability is comparable with conventional techniques. Aim: To report short- and long-term results with the use of a robot-assisted transthoracic approach. Patients and Methods: Review of patients undergoing heart surgery between 2015 and 2019 using a robot assisted minimally invasive technique in a single center. We analyzed demographic characteristics, surgical and early ultrasound results. Results: Thirteen procedures were reviewed, nine mitral valve repairs (MVR) in patients aged $61 \pm 21$ years (seven males) and four atrial septal defect (ASD) closures in patients aged from 24 to 52 years (three men). For $M V R$, the average extracorporeal circulation and myocardial ischemia times were $120 \pm 20.9$ and $89 \pm 21$ minutes, respectively. The median hospitalization was four days. Two cases of MVR had postoperative complications. There was no mortality. All cases showed improvement in their symptoms. Ultrasound findings showed no postoperative mitral insufficiency except in one case. Conclusions: We report very good results in both complex mitral repair and CIA closure, comparable to centers with high standards in minimally invasive robot-assisted heart surgery.

(Rev Med Chile 2019; 147: 1303-1307)

Key words: Heart Septal Defects, Atrial; Mitral Valve; Prolapse; Robotics.

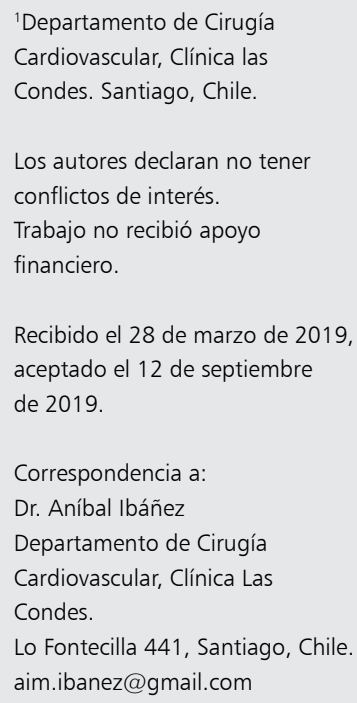

L a cirugía cardíaca valvular es un procedimiento complejo, que generalmente se $\checkmark$ realiza a través de una esternotomía media convencional para lograr una excelente exposición. El abordaje mínimamente invasivo debido a sus buenos resultados, ha permitido el desarrollo de procedimientos quirúrgicos en un espacio reducido, a través de pequeñas incisiones. Durante las últimas décadas, las mejoras en los equipos y técnicas endoscópicas, además de los avances en perfusión cardiopulmonar con la utilización de cánulas endovasculares especializadas, la visualización intracardíaca, instrumentalización y la telemanipulación robótica han tenido un cambio hacia una cirugía cardíaca eficiente y segura. Por primera vez en 1996, se utilizaron mini-esternotomías, incisiones paraesternales y minitoracotomías para iniciar operaciones valvulares ${ }^{1,2}$. Arom, Gundry y Cosgrove ${ }^{3-6}$ fueron los primeros en realizar cirugía válvular mitral con pequeñas incisiones y baja mortalidad. Carpentier, realizó la primera RM utilizando un robot da $\mathrm{Vinci}^{4}$ con buenos resultados. La cirugía cardíaca robótica es un abordaje minimamente invasivo, que está ampliamente demostrado por la literatura ${ }^{1-4} y$ ha logrado buenos resultados debido a la eliminación de las poten- 
ciales complicaciones de la esternotomía, baja tasa infección, menor necesidad de tranfusiones y readmisión, logrando disminuir la estadía hospitalaria y acortar los tiempos de recuperación. Además, éstos beneficios se acentúan en pacientes que han tenido cirugía cardíaca previa, ya que elimina los riesgos de una re-esternotomía ${ }^{5-9}$. No obstante, las principales limitaciones están relacionadas con la calcificación anular mitral, la aterosclerosis aórtoiliaca, y la insuficiencia aórtica ${ }^{10}$. Los avances tecnológicos han facilitado la reparación de condiciones cardíacas estructurales complejas que incluyen la plastía de válvula mitral y tricuspídea, el cierre de defecto septal atrial y foramen oval permeable, además de remover tumores cardíacos, y la cirugía de revacularización coronaria que actualmente se encuentra en desarrollo ${ }^{12,14}$. El programa de cirugía cardíaca robótica comenzó en nuestro centro en 2015, y nuestro objetivo es mostrar los procedimientos quirúrgicos cardíacos asistidos por robot evaluando los resultados a corto y largo plazo.

\section{Paciente y Método}

Se seleccionaron todos los pacientes con diagnóstico único de insuficiencia mitral (IM) severa o comunicación interauricular (no susceptible de manejo percutáneo), que fueron reparados por vía mínimamente invasiva asistido por robot, durante diciembre de 2015 a febrero de 2019. El diagnóstico se realizó con la historia clínica, examen físico y ecografía cardíaca transtorácico (ETT). El tipo de RM fue definida según los hallazgos anátomo-funcionales de la válvula bajo visión ecográfica transesofágico (ETE). La totalidad de los procedimientos fueron asisitidos vía robot da Vinci SI, con anestesia general e intubación endotraqueal de doble lumen. El paciente es posicionado en semi-decúbito lateral izquierdo $\left(30^{\circ}\right)$, se utiliza una cánula arterial \#17 en posición yugular interna derecha para drenaje de vena cava superior y una cánula venosa multifenestrada femoral derecha para el drenaje de la vena cava inferior, en ambos casos, se confirma con ETE el adecuado posicionamiento de las cánulas en la unión cavo-atrial. El bypass cardiopulmonar es periférico vía femoral con clampeo aórtico transtorácico y cardioplejía anterógrada Del Nido. El abordaje utilizado fue a través de tres puertos transtorácicos asociado a una incisión intercostal derecha de $4 \mathrm{~cm}$. Se confecciona anuloplastía con anillo flexible en todos los casos (Figura 1). En los 2 primeros pacientes se utiliza sutura continua intracorpórea descrita por Mihaljevic ${ }^{12}$, y en el resto de los pacientes LSI COR-KNOT ${ }^{\circledR}$, sistema rápido y automático de fijación de la sutura, permitiendo acortar los tiempos operatorios ${ }^{13}$. Se analizaron aspectos demográficos, tiempos quirúrgicos, horas de ventilación mecánica, días de UCI y alta médica, además
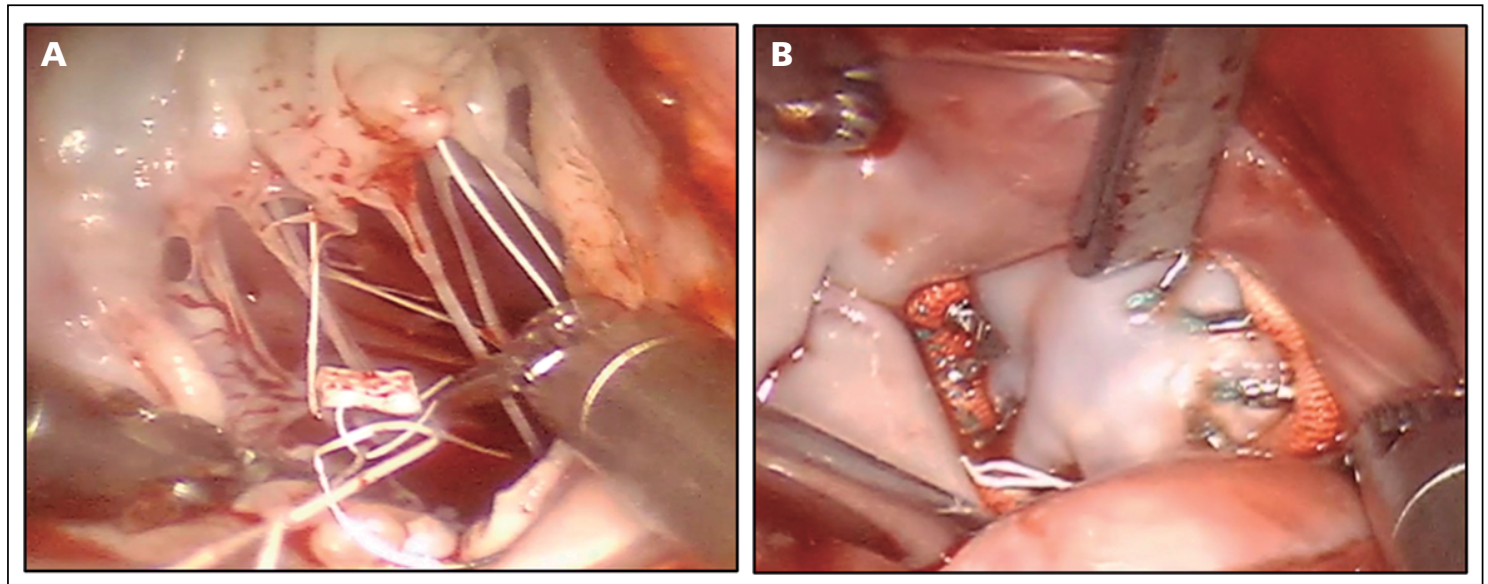

Figura 1. Imágenes intraoperatorias mostrando la visualización de la válvula mitral utilizando la plataforma del robot da Vinci. (A) Ambos brazos del robot demuestran el proceso de plastía mitral con neocuerdas de Gore-Tex en el segmento patológico del velo anterior de la válvula mitral para reestablecer la competencia valvular. (B) La válvula mitral completamente reparada con asistencia robótica da Vinci. Un anillo ha sido utilizado con prueba hídrica satisfactoria. 
de la morbilidad y resultados ecocardiográficos al mes de control post operatorio. El proyecto fue aprobado por el Comité Ético-Científico de Clínica Las Condes.

\section{Resultados}

Todos los pacientes seleccionados fueron operados. Un total de 13 casos, 9 corresponden a reparaciones de válvula mitral $(\mathrm{RM})$ y 4 a cierre de CIA. Los 4 pacientes con CIA tipo Ostium Secundum, corresponden a tres hombres de 24, 36 y 52 años, y una mujer de 29 años. El primer caso se reparó con parche de pericardio bovino y los otros tres con matriz extracelular CorMatrix ${ }^{\circledR 11}$. El tiempo promedio de circulación extracorpórea fue $125 \mathrm{~min}$ (rango de 120 a140 min), y el clamp aórtico fue de $85 \mathrm{~min}$ (rango de 80 a $112 \mathrm{~min}$ ). El estudio con ETE intraoperatoria, mostró ausencia de shunt interauricular. Todos egresaron al cuarto día, y no hubo complicaciones. Al control postoperatorio del mes, presentaron remisión completa de los síntomas, mejoría de clase funcional (CF) de New York Heart Association (NYHA) de tipo II a tipo I , la ETT sin signos de CIA y normalización de cavidades derechas, además de disminución de hipertensión pulmonar de moderada a leve. La RM se realizó en 7 hombres y 2 mujeres con edad promedio de 61 años $\pm 20,8$ años. Todos lo pacientes estaban sintomáticos (Tabla 1). Se realizaron distintas reparaciones de la válvula mitral. Tres pacientes con resección triangular de $\mathrm{P} 2$ debido a flail y prolapso, un caso con cuadrantectomía de P2 por enfermedad de Barlow y cinco pacientes con reparación sin técnica resectiva, cuatro plastías del velo anterior con cuerdas de politetrafluoroetileno (PTFE) y solo un caso con anuloplastía por dilatación del anillo valvular y leve prolapso de P2. Se utilizó anillo flexible incompleto (Duran Band número $33 \mathrm{~mm}$, Medtronic $\left.{ }^{\circledR}\right)$. El tiempo de circulación extracorpórea fue $120 \pm 20,9 \mathrm{~min}$ y clamp aórtico de $89 \pm 21 \mathrm{~min}$. La mediana de ventilación mecánica fueron $6 \mathrm{~h}$. Se utilizaron 3 unidades de hemoderivados en el postoperatorio. La duración de la estadía en unidad de cuidados intensivos y de hospitalización fueron de una mediana de uno y cinco días, respectivamente. Dos pacientes presentaron egreso prolongado de $12 \mathrm{y}$ 25 días, el primero debido a una neumonía post operatoria, y el segundo debido a su alta comorbi- lidad. Tuvimos un caso con fibrilación auricular de Novo. No hubo mortalidad ni reoperación. En el seguimiento ecográfico al primer y tercer mes, no presentaron IM postoperatoria, salvo un caso de

Tabla 1. Características de los pacientes $(\mathbf{n}=\mathbf{9})$

\begin{tabular}{|lc|}
\hline Características & $\begin{array}{c}\text { n de casos } \\
\text { o promedio } \\
\pm \text { DE }\end{array}$ \\
\hline $\begin{array}{l}\text { Edad (años) } \\
\text { Masculino }\end{array}$ & $\begin{array}{c} \pm 20,8 \\
\text { Índice de superficie corporal }\end{array}$ \\
Clase funcional NYHA & $1,81 \pm 0,2$ \\
I-II & \\
III-IV & 2 \\
Fracción de eyección & 7 \\
Comorbilidades cardíacas & $56 \pm 5 \%$ \\
Fibrilación auricular & \\
Infarto al miocardio previo & 3 \\
Insuficiencia tricuspídea leve & 0 \\
Insuficiencia aórtica leve & 1 \\
Comorbilidades no cardíacas & 1 \\
Enfermedad carotídea asintomática & 1 \\
Hipertensión & 2 \\
Enfermedad pulmonar obstructiva crónica & 2 \\
\hline
\end{tabular}

NYHA: New York Heart Association. DE: Desviación Estándar.

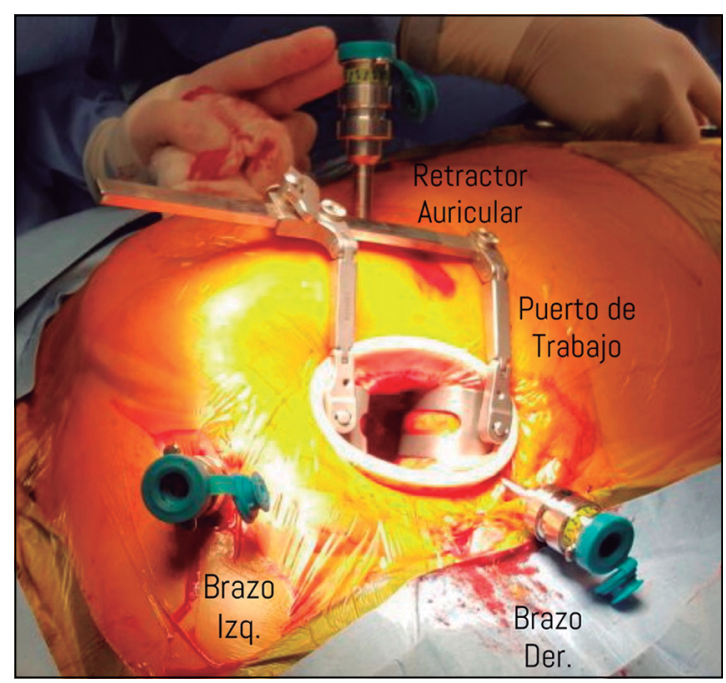

Figura 2. Puertos están en posición como también el retractor de finochietto. 
Tabla 2. Detalle operatorio

\begin{tabular}{|lc|}
\hline Detalle & n de casos \\
\hline Resección triangular de velo posterior & 3 \\
\hline Resección cuadrangular de velo posterior & 1 \\
Cuerda artificial PTFE en velo anterior & 4 \\
Anuloplastía con anillo flexible & 1 \\
\hline
\end{tabular}

PTFE: politetrafluoroetileno.

Tabla 3. Resultados perioperatorios

\begin{tabular}{|lc|}
\hline Variables & Resultados \\
\hline Tiempo de circulación extracorpórea (min) & $120 \pm 20,9$ \\
\hline Tiempo de clamp aórtico (min) & $89 \pm 21$ \\
\hline Horas de ventilación mecánica (mediana) & 6 \\
\hline Transfusión hemoderivados perioperatorio ( $\mathrm{n})$ & 3 \\
\hline Día(s) de UCl (mediana) & 1 \\
Mortalidad operatoria (n) & 0 \\
\hline ACV permanente (n) & 0 \\
\hline Infección de herida esternal (n) & 0 \\
Reoperación por cualquier causa (n) & 0 \\
Ventilación mecánica prolongada (n) & 0 \\
Fibrilación auricular de novo (n) & 1 \\
Isquemia aguda (n) & 0 \\
\hline Falla renal con hemodiálisis (n) & 0 \\
Disección de aorta (n) & 0 \\
Implante de marcapaso (n) & 0 \\
Duración de hospitalización (mediana en días) & 5 \\
\hline
\end{tabular}

UCl: unidad de cuidados intensivos.

grado leve. La mediana de seguimiento fueron 30 meses, todos los pacientes se mantienen en control y seguimiento, con mejoría de CF a tipo I-II, y sin signos ecográficos de insuficiencia mitral, salvo en un caso de grado leve.

\section{Discusión}

La reparación minimamente invasiva asistida por robot, tanto en CIA y RM es una técnica segura y efectiva. Se realizó sin mortalidad, mínima morbilidad y sin casos de conversión. En el egreso hospitalario solo un paciente tuvo un grado de insuficiencia mitral, que fue leve. El seguimiento y los resultados a largo plazo de nuestros pacientes muestran sobrevida y durabilidad de la reparación similar a abordajes convencionales ${ }^{7,15}$, y es comparable a centros con altos estándares de cirugía cardíaca mínimamente invasiva asistida por robot ${ }^{16}$. La complejidad del abordaje robótico se atribuye principalmente a una mayor curva de aprendizaje debido a que además, requiere experiencia en el manejo de esta patología por vía convencional. Una de las controversias que se han generado en relación a esta técnica es el factor costo. Claramente encontramos que la aplicación de la cirugía robótica aumenta los costos hospitalarios marginalmente, mientras que los disminuye con la menor estadía hospitalaria y tiempo de retorno al trabajo. A pesar de no haber sido discutido en nuestro trabajo, ha sido ampliamente mostrado tanto en grandes centros como en nuestra institución, que el costo es similar al abordaje convencional ${ }^{17-19}$. Un adecuado estudio preoperatorio es fundamental para la selección de los pacientes y lograr excelentes resultados.

\section{Referencias}

1. Mohr FW, Falk V, Diegeler A, Walther T, Gummert JF, Bucerius J, et al. Computer-enhanced "robotic" cardiac surgery: experience in 148 patients. J Thorac Cardiovasc Surg 2001; 121 (5): 842-53.

2. Chitwood WR Jr, Rodríguez E, Chu MW, Hassan A, Ferguson TB, Vos PW, et al. Robotic mitral valve repairs in 300 patients: a single-center experience. J Thorac Cardiovasc Surg 2008; 136 (2): 436-41.

3. Suri RM, Dearani JA, Mihaljevic T, Chitwood WR Jr, Murphy DA, Trento A, et al. Mitral valve repair using robotic technology: Safe, effective, and durable. J Thorac Cardiovasc Surg 2016; 151 (6): 1450-4.

4. Carpentier A, Loulmet D, Aupecle B, Kieffer JP, Tournay D, Guibourt P, et al. Computer assisted open-heart surgery. First case operated on with success. C R Acad Sci III 1998; 321 (5): 437-42.

5. Suri RM, Burkhart HM, Daly RC, Park SJ, Sundt TM, $\mathrm{Li} Z$, et al. Robotic mitral valve repair for all prolapse subsets using techniques identical to open valvuloplasty: establishing the benchmark against which percutaneous interventions should be judged. J Thorac Cardiovasc Surg 2011; 142: 970-9.

6. Paul S, Isaacs AJ, Jalbert J, Osakwe NC, Salemi A, Girar- 
di LN, et al. A population-based analysis of robotic-assisted mitral valve repair. Ann Thorac Surg 2015; 99: 1546-53.

7. Gammie JS, Zhao Y, Peterson ED, O’Brien SM, Rankin JS, Griffith BP. Less-invasive mitral valve operations: trends and outcomes from the Society of Thoracic Surgeons Adult Cardiac Surgery Database. Ann Thorac Surg 2010; 90: 1401-8.

8. Nifong LW, Chu VR, Bailey BM, Maziarz DM, Sorrell VL, Holbert D, et al. Robotic mitral valve repair: experience with the da Vinci system. Ann Thorac Surg 2003; 75 (2): 438-43.

9. Ishikawa N, Watanabe G. Robot-Assisted Cardiac Surgery. Ann Thorac Cardiovasc Surg 2015; 21 (4): 322-8.

10. Gillinov A, Suri R, Mick S, Mihaljevic T. Robotic mitral valve surgery: current limitations and future directions. Ann Cardiothorac Surg 2016; 5: 573-6.

11. Holubec T, Caliskan E, Sundermann S, Starck CT, Plass A, Bettex D. The Use of Extracellular Matrix Patches in Cardiac Surgery Card Surg 2015; 30: 145-8.

12. Mihaljevic T, Jarrett C, Gillinov M, Blackstone E. A novel running annuloplasty suture technique for robotically assisted mitral valve repair. The Journal of Thoracic and Cardiovascular Surgery 2010; 139 (5): 1343-4.

13. Sabik JF, Raza S, Chavin KD. Safety and benefits of new techniques and technologies in less invasive mitral valve repair. J Card Surg 2018; 33(10): 609-19.
14. Suri RM, Taggarse A, Burkhart HM, Daly RC, Mauermann W, Nishimura RA, et al. Robotic Mitral Valve Repair for Simple and Complex Degenerative Disease: Midterm Clinical and Echocardiographic Quality Outcomes. Circulation 2015; 132 (21): 1961-8.

15. Murphy DA, Moss E, Binongo J, Miller JS, Macheers S, Sarin EL, et al. The Expanding Role of Endoscopic Robotics in Mitral Valve Surgery: 1,257 Consecutive Procedures. Ann Thorac Surg 2015; 100 (5): 1675-81; discussion 1681-2.

16. Mihaljevic T, Jarrett CM, Gillinov AM, Williams SJ, DeVilliers PA, Stewart WJ, et al. Robotic repair of posterior mitral valve prolapse versus conventional approaches: potential realized. J Thorac Cardiovasc Surg 2011; 141 (1): 72-80.

17. Javadikasgari H, Suri M, Tappuni B, Lowry A, Mihaljevic $\mathrm{T}$, Mick $\mathrm{S}$, et al. Robotic mitral valve repair for degenerative posterior leaflet prolapse. Ann Cardiothorac Surg 2017; 6 (1): 27-32.

18. Mihaljevic T, Koprivanac M, Kelava M, Goodman A, Jarrett C, Williams SJ, et al. Value of robotically assisted surgery for mitral valve disease. JAMA Surg 2014; 149: 679-86.

19. Suri RM, Thompson JE, Burkhart HM, Huebner M, Borah BJ, Li Z, et al. Improving affordability through innovation in the surgical treatment of mitral valve disease. Mayo Clin Proc 2013; 88: 1075-84. 